\title{
Correlation of modified CT Severity Index with Complications of Acute Pancreatitis
}

\author{
Anuradha Kapali ${ }^{1}$ \\ ${ }^{1}$ Associate Professor, Kidwai Cancer Institute, Bangalore, India \\ Corresponding author: Anuradha Kapali, Bhoopalm, 4th Main Raj Kumar Layout, Kalkere Main Road, Horamavu, \\ Bangalore.560043, India
}

DOI: http://dx.doi.org/10.21276/ijcmsr.2019.4.4.27

How to cite this article: Anuradha Kapali. Correlation of modified CT Severity Index with complications of acute pancreatitis. International Journal of Contemporary Medicine Surgery and Radiology. 2019;4(4):D113-D116.

\section{A B S T R A C T}

Introduction: Acute pancreatitis is a significant cause of morbidity and mortality and is known to run an unpredictable course. Severe pancreatitis occurs in $20 \%-30 \%$ of all patients with acute pancreatitis and is characterized by a protracted clinical course, multiorgan failure, and pancreatic necrosis. Modified Computed Tomography Severity Index (MCTSI) is used as a scoring system in acute pancreatitis. This study aims to correlate the MCTSI grading system with patient outcome in terms of organ failure, mortality and duration of hospital stay.

Materials and methods: This was a prospective study conducted for a period of two years. 39 Cases diagnosed as acute pancreatitis were included in this study. These patients underwent contrast enhanced computed tomography (CECT) of the abdomen and pelvis. The findings were recorded as per the modified CT severity index.

Results: There was significant correlation between necessity of ICU admission and duration of ICU stay and grade of pancreatitis, positive correlation with duration of ward stay and strong correlation with total duration of hospital stay. Higher CT grade is positively associated with development of local complications and systemic complications.

Conclusion: Grading by modified CT severity index has a significant correlation with necessity of ICU admission, duration of ICU stay and total duration of hospital stay. Modified CT severity index can be used to predict the possibility of developing local and systemic complications, the need for interventions and necessity of ICU admission.

Keywords: Acute Pancreatitis, Modified CT Severity Index, ICU Admission, Local, Systemic Complications. Prognosis.

\section{INTRODUCTION}

Acute pancreatitis is a significant cause of morbidity and mortality and is known to run an unpredictable course. Severe pancreatitis occurs in $20 \%-30 \%$ of all patients with acute pancreatitis and is characterized by a protracted clinical course, multiorgan failure, and pancreatic necrosis. Individual laboratory indexes (markers of pancreatic injury, markers of inflammatory response), while promising, have not yet gained clinical acceptance. Numeric grading systems like RANSON and APACHE II are commonly used today as indicators of disease severity. While RANSON score cannot be used for the first $48 \mathrm{hrs}$, APACHE score is cumbersome to use.

CT (Computed tomography) severity index was used initially which was popularly called Balthazar scoring system. Now Modified Computed Tomography Severity Index (MCTSI) has been introduced which differs from the Computed Tomography Severity Index (CTSI) by including the presence of extra pancreatic complications and grading the peripancreatic fluid collection in terms of presence or absence instead of the number of fluid collections. The grading of necrosis is also different in this system.

This study aimed to correlate the MCTSI grading system with patient outcome in terms of organ failure, mortality and duration of hospital stay.

\section{MATERIAL AND METHODS}

This was a prospective study conducted for a period of two years. 39 Cases diagnosed as acute pancreatitis based on history and serum amylase and lipase levels were included in this study. These patients underwent contrast enhanced computed tomography (CECT) of the abdomen and pelvis. Pre contrast and post contrast study were performed from the chest base to the pubic symphysis with $5 \mathrm{~mm}$ slice thickness. The findings were recorded as per the modified CT severity index. Percentage necrosis was calculated by inbuilt software.

Patients with chronic pancreatitis and patients who are discharged from hospital before they were stabilized or discharged against medical advice were excluded from the study.

Ethical clearance was obtained from the institution.

The significance of correlation between modified CT severity index and necessity of ICU admission, duration of ICU stay and total duration of hospital stay was calculated in terms of $P$ value. The accuracy of modified CT severity index in predicting local and systemic complications was calculated in terms of sensitivity, specificity, positive predictive value and negative predictive value. 


\section{RESULTS}

The patients with acute pancreatitis were graded according to MCTSI (table 1). The scores are assigned for pancreatic inflammation, pancreatic necrosis and extra pancreatic complications and added up to give the final score. They were classified into mild (grade 2 and 4), moderate (grade 6) and severe pancreatitis (grade 8 and 10), and were followed up for presence of systemic complications (shock, abdominal hemorrhage, renal failure, respiratory failure) local complications (pseudocyst formation, abscess, vascular complications), their duration of hospital stay (ward and ICU), necessity for ICU (Intensive care unit) admission and necessity for interventions.

The distribution of patients in our study according to the MCTSI, necessity of ICU admission and the development of local and systemic complications are given in table 2 . The duration of ICU, ward and total stay is given in table 3.

There was significant correlation between necessity of ICU admission and grade of pancreatitis. There is significant correlation with duration of ICU stay and grade of pancreatitis $(P=0.006)$, positive correlation with duration of ward stay $(P=0.010)$, and strong correlation with total duration of hospital stay $(P=0.004)$. Higher CT grade is

\begin{tabular}{|l|c|}
\hline Prognostic Indicator & Points \\
\hline Pancreatic inflammation & 0 \\
\hline Normal pancreas & 2 \\
\hline $\begin{array}{l}\text { Intrinsic pancreatic abnormalities with or without } \\
\text { inflammatory changes in peripancreatic fat }\end{array}$ & 4 \\
\hline $\begin{array}{l}\text { Pancreatic or peripancreatic fluid collection or peri- } \\
\text { pancreatic fat necrosis } \\
\text { Pancreatic necrosis }\end{array}$ & 0 \\
\hline None & 2 \\
\hline$\leq 30 \%$ & 4 \\
\hline$>30 \%$ & 2 \\
\hline $\begin{array}{l}\text { Extrapancreatic complications (one or more of } \\
\text { pleural effusion, ascites, vascular complications, } \\
\text { parenchymal complications, or gastrointestinal tract } \\
\text { involvement) }\end{array}$ & \\
\hline \multicolumn{1}{|c|}{ Table-1: Modified CT Severity Index } \\
\hline
\end{tabular}

positively associated with presence of pseoducyst $(P=0.143)$, abscess $(P=0.179)$ and local complications $(P=0.143)$. Higher CT grade is significantly associated with presence of systemic complications with $P=0.028$.

\section{DISCUSSION}

Contrast-enhanced CT is the imaging modality of choice for the diagnosis and staging of acute pancreatitis. ${ }^{1} \mathrm{We}$ evaluated our cases with CECT and correlated the findings with patient outcome.

The total age range of patients was from 4 years to 67 years. No association of age and gender was noted with severity of pancreatitis in our study. These observations was similar to that of a study conducted by Lankish et al. ${ }^{2}$

The CT grades were classified into 2, 4, 6, 8 and 10 according to the MCTSI. We further classified the grades into mild (grade 2 and 4), moderate (grade 6) and severe (grade 8 and 10). The previous studies by Bollen et $\mathrm{al}^{3}$ and Mortele et $\mathrm{al}^{4}$ have classified grade 2 as mild, grade 4 and 6 as moderate and grade 8 and 10 as severe. The prognosis of patients with grade 2 and 4 pancreatitis was similar and milder than patients who had a grade of 6 as observed in our study, hence were grouped together in our study.

ICU admission was needed for $51 \%$ patients with pancreatitis.

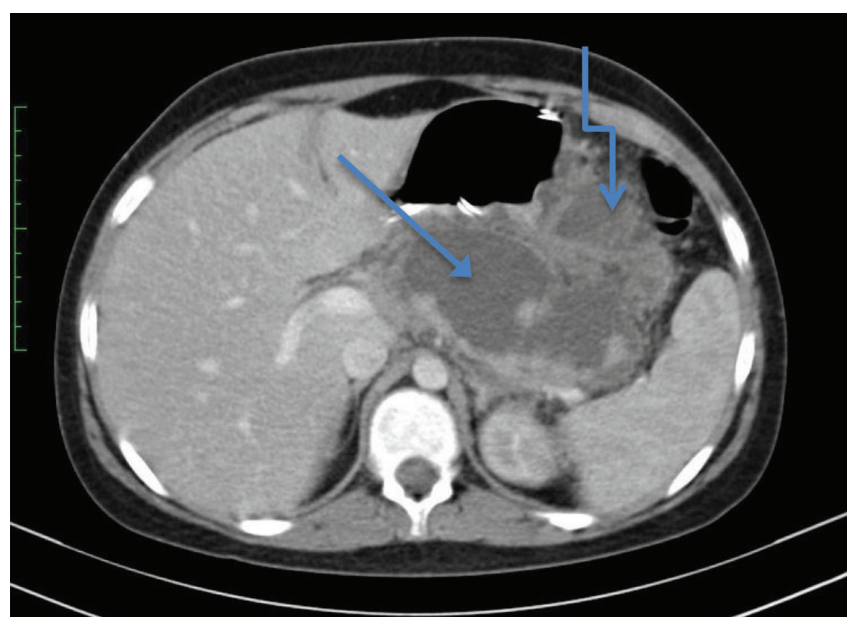

Figure-1: Pancreatic necrosis (straight arrow) with acute fluid collection (elbow arrow): grade 10 pancreatitis.

\begin{tabular}{|l|c|c|c|c|c|c|}
\hline CT grade & $\begin{array}{c}\text { Number of } \\
\text { patients }\end{array}$ & $\begin{array}{c}\text { ICU admission } \\
\text { needed }\end{array}$ & $\begin{array}{c}\text { Pseudocyst } \\
\text { fromation }\end{array}$ & $\begin{array}{c}\text { Abscess } \\
\text { formation }\end{array}$ & $\begin{array}{c}\text { Intervention } \\
\text { Necessity }\end{array}$ & $\begin{array}{c}\text { Systemic } \\
\text { complication }\end{array}$ \\
\hline 2 & 6 & $2(33.3 \%)$ & 0 & 0 & 0 & 0 \\
\hline 4 & 7 & $1(14.2 \%)$ & 0 & 0 & 0 & 0 \\
\hline 6 & 19 & $10(62.5 \%)$ & $6(66.7 \%)$ & 0 & 0 & 0 \\
\hline 8 & 2 & $2(100 \%)$ & $1(11.1 \%)$ & 0 & 0 & 0 \\
\hline 10 & 5 & $5(100 \%)$ & $2(22.2 \%)$ & 1 & 2 & $2(100 \%)$ \\
\hline \multicolumn{2}{|r|}{ Table-2: Distribution of CT grade and Correlation of CT grade with development of local and systemic complication } \\
\hline
\end{tabular}

\begin{tabular}{|l|c|c|c|}
\hline CT grade & ICU (days) & WARD (days) & Hospital stay in days \\
\hline 2 and 4 & $0.47 \pm 0.97$ & $6.77 \pm 2.52$ & $7.23 \pm 2.77$ \\
\hline 6 & $2.58 \pm 3.70$ & $9.21 \pm 4.37$ & $11.78 \pm 6.29$ \\
\hline 8 and 10 & $12.86 \pm 18.47$ & $16.00 \pm 12.43$ & $28.86 \pm 29.59$ \\
\hline P value & $0.006^{* *}$ & $0.010^{*}$ & $0.004^{* *}$ \\
\hline \multicolumn{2}{|r|}{} \\
\hline
\end{tabular}


Patients with a mild grade needed ICU admission in an average of $23 \%$, moderate grade needed admission in $62 \%$ and severe grade needed admission in $100 \%$ of patients. There is significant correlation with duration of ICU stay and grade of pancreatitis too. Patients with a mild grade had a mean duration of stay in ICU for 0.4 days, moderate grade had duration of stay of 2.5 days and with a severe grade of pancreatitis had duration of stay of 12.8 days.

Strong correlation was seen between patient's CT grade and total duration of hospital stay. Mean duration of stay was 7.2 days in mild, 11.7 days in moderate and 28.8 days in severe pancreatitis. A study by Mortele et al. ${ }^{4}$ (published in 2004) showed a significant correlation between MCTSI grade of pancreatitis and length of hospital stay.

The local complications identified in the study were pseudocysts and abscess formation, one patient developed splenic artery aneurysm. Pseudocyst was seen in 9 patients (23\%) in our study. Pseudocyst formation occurred in $50 \%$ of patients in a study conducted by Gonzalez et al. ${ }^{5}$ Abscess was detected in 1 patient (2.6\%). Presence of local complications was positively associated with CT grading. No local complications were seen in patients with mild pancreatitis. About $31 \%$ of patients with moderate pancreatitis and $42 \%$ of patients with severe pancreatitis had developed local complications.

In our study intervention was needed in form of surgical debridement in one patient with grade 10 (figure 1) of AP (Acute pancreatitis). Radiological intervention was needed in 2 patients (5\%). Aspiration of pseudocyst was needed in one patient with grade 6 of pancreatitis and embolisation of gastroduodenal and splenic arteries was needed in one patient with grade $10 \mathrm{AP}$. Thus patients who need an intervention have more severe CT grades. This is similar to the study by Bollen et $\mathrm{al}^{3}$ which demonstrated that development of local complications and need for intervention was significantly associated with grade of pancreatitis.

Systemic complication developed in 2 patients (5\%). The complications seen were shock and abdominal hemorrhage. Systemic complications were seen only in patients with a CT grade of 10, which showed a significant association. Significant association was noted between development of systemic complications and grading of AP by Bollen et al. ${ }^{3}$ Maximum patients who developed systemic complications were in severe grade.

In our study pancreatic necrosis was identified in 7 patients (18\%). No necrosis was seen in 32 patients (82\%). A study by Bollen et al and Mortele et al identified necrosis in 18\% and $15 \%$ of patients with acute pancreatitis respectively. Necrosis occurs early, within the first 24-48 hours, and with few exceptions remains stable during a given episode of acute pancreatitis. ${ }^{6}$

No mortality due to pancreatitis was observed in our study. In the study by Bollen et al mortality was seen in $6 \%$ of patients and in $1.5 \%$ patients in the study by Mortele et al.

The accuracy of MCTSI in predicting the systemic complications in our study was as were as follows: Sensitivity $=100 \%$, Specificity $=91.8 \%$, Positive predictive value $=40 \%$, Negative predictive value $=100 \%$, Prevalence of systemic complications in patients with acute pancreatitis $=5 \%$.
The accuracy of MCTSI in predicting the local complications were as follows: Sensitivity $=100 \%$, Specificity $=43 \%$, Positive predictive value $=34.6 \%$, Negative predictive value $=100 \%$, Prevalence of local complications in patients with acute pancreatitis $=23 \%$.

The accuracy of MCTSI in predicting the necessity of ICU were as follows: Sensitivity $=76.9 \%$, Specificity $=52 \%$, Positive predictive value $=52.6 \%$, Negative predictive value $=76.9 \%$. All patients with grade 8 and grade 10 needed ICU admission.

Diagnosis on basis on clinical evaluation is missed in 30\%$40 \%$ of patients with fatal necrotizing pancreatitis until the time of autopsy. Thus clinical signs have only limited value for the assessment of the severity of acute pancreatitis. ${ }^{7}$

In predicting the prognosis, there are several scoring indices that have been used as predictors of survival. Two such scoring systems are the Ranson criteria and APACHE II (Acute Physiology and Chronic Health Evaluation) indices. Most studies report that the Apache score may be more accurate. ${ }^{8,9}$ The Apache II can be fully calculated upon admission. However the Apache II is more cumbersome to calculate.

Ranson signs have been used for many years to assess severity of acute pancreatitis but have the disadvantage of requiring a full $48 \mathrm{~h}$ for a complete evaluation. In general, when Ranson signs are $<3$, mortality is $0-3 \%$; when $\geq 3,11-15 \%$; when $\geq 6$, $40 \% .{ }^{10}$ However, a more recent comprehensive evaluation of 110 studies concluded that Ranson signs provided very poor predictive power of severity of acute pancreatitis. ${ }^{11}$

Higher APACHE II is associated with a higher mortality $(<4 \%$ with an APACHE-II $<8$ and $11-18 \%$ with an APACHE-II >8). The advantage of the APACHE-II score was the availability of this information within the first 24 $\mathrm{h}$ and daily. An APACHE-II score that increases during the first $48 \mathrm{~h}$ is strongly suggestive of the development of severe pancreatitis, whereas an APACHE-II that decreases within the first $48 \mathrm{~h}$ strongly suggests mild pancreatitis. The sensitivity and specificity of the APACHE II system for assessing severe acute pancreatitis at the time of admission are $75 \%$ and $79 \%$, respectively. ${ }^{12}$

Developed in the early 1990s by Emil J. Balthazar et al. the Computed Tomography Severity Index (CTSI) is a grading system used to determine the severity of acute pancreatitis. CTSI's staging of acute pancreatitis severity has been shown by a number of studies to provide more accurate assessment than APACHE II, Ranson, and C reactive protein C-reactive protein(CRP) level. ${ }^{13}$

The modified CT severity index correlated more closely with patient outcome measures than the currently accepted CT severity index, with similar interobserver variability. ${ }^{4}$

According to Bollen et $\mathrm{al}^{3}$ when comparing the MCTSI and APACHE (Acute Physiology and Chronic Health Evaluation) II, no statistically significant difference was found for mortality, ICU stay and organ failure. The MCSTI accurately correlated with pancreatic infection and the need for intervention compared with APACHE II. CT is the modality of choice for detecting the local complications. Thus the MCTSI is as useful as APACHE II in predicting the severity of AP in terms of ICU stay and 
organ failure and is better than APACHE II in detecting the local complications and confirming necrosis in AP. But MCTSI has a disadvantage that the study cannot be carried out within $48 \mathrm{hrs}$ as this is the time taken to demonstrate necrosis. However APACHE II score can be calculated even at the time of admission.

The limitations of the study are as follows:

- Nonrandomized study.

- Patients with mild acute pancreatitis could not be included in the present study as CT scan is not indicated in all cases of pancreatitis.

- Pancreatitis patients with renal failure were excluded in the study as contrast

- administration is contraindicated in them.

- The small sample size.

\section{CONCLUSION}

Grading by modified CT severity index has a significant correlation with necessity of ICU admission, duration of ICU stay and total duration of hospital stay. Modified CT severity index can be used to predict the possibility of developing local and systemic complications, the need for interventions and necessity of ICU admission.

\section{REFERENCES}

1. Scaglione M, Casciani E, Pinto A, Andreoli C, De Vargas M, Gualdi GF. Imaging assessment of acute pancreatitis: a review. Semin Ultrasound CT MR. 2008;29 (5): 322-340.

2. Lankisch D, Paul G, Burchard R, Petersen S. Etiology and Age Have Only a Limited Influence on the Course of Acute Pancreatitis. Pancreas. 1996;13(4):344-349.

3. Bollen T, Singh V, Maurer R. Comparative Evaluation of the Modified CT Severity Index and CT Severity Index in Assessing Severity of Acute Pancreatitis. AJR. 2011;197(3):386-392.

4. Mortele K, Wiesner W, Intriere L, Shankar S, Kelly H. A Modified CT Severity Index for Evaluating Acute Pancreatitis: Improved Correlation with Patient Outcome. AJR. 2004;183(5):1261-1265.

5. Gonzale P, Nagar B, Gorelick S. Pseudocyst formation in acute pancreatitis. AJR. 1976;127(6):315-317.

6. Balthazar E. Acute Pancreatitis: Assessment of Severity with Clinical and CT Evaluation. Radiology. 2002;223(1):603-613.

7. Corfield AP, Cooper MJ, Williamson RCN. Prediction of severity in acute pancreatitis: prospective comparison of three prognostic indices. Lancet. 1985;2(5):403-407.

8. Larvin M, McMahon M. APACHE-II score for assessment and monitoring of acute pancreatitis. Lancet 2. 1989;6(1):201-5.

9. Yeung Y, Lam B, Yip A. APACHE system is better than Ranson system in the prediction of severity of acute pancreatitis. Hepatobiliary Pancreat Dis Int. 2009;5(2):294-9.

10. Blum T, Maisonneuve P, Lowenfels AB. Fatal outcome in acute pancreatitis: Its occurrence and early prediction. Pancreatology. 2001;1(5):237-41.

11. De Bernardinis M, Violi V, Roncoroni L. Discriminant power and information content of Ranson's prognostic signs in acute pancreatitis: A meta-analytic study. Crit Care Med. 1999;27(1):2272-83.

12. Osvaldt AB, Viero P, BorgesMS, Wendt LR, Bersch VP, Rohde L. Evaluation of Ranson, Glasgow, APACHEII, and APACHE-O criteria to predict severity in acute biliary pancreatitis. Int Surg. 2001;86(4):158-161.

13. Triantopoulou C, Lytras D, Maniatis P, Chrysovergis D, Manes K, Siafas I. Computed tomography versus Acute Physiology and Chronic Health Evaluation II score in predicting severity of acute pancreatitis: a prospective, comparative study with statistical evaluation. Pancreas. 2007;35 (3):238-242.

Source of Support: Nil; Conflict of Interest: None

Submitted: 15-10-2019; Accepted: 16-11-2019; Published online: 15-12-2019 